\title{
G305.8 - 0.2: A YOUNG OBJECT WITH A DUST AND GAS ENVELOPE
}

\author{
J.W.S. Vilas Boas, E. Scalise Jr॰ (1), G.G. Sanzovo(2), \\ G. Mendes Pacheco(1), A.M. Gómez Balboa (3) \\ CRAAE - EPUSP/PTR, CP 8174, 05508 S.Paulo, SP; \\ (1) SCT - INPE - DAS, CP 515, 12201 S.J.Campos, SP; \\ (2) Univ. Est. de Londrina, Depart. de Fisica, 86020 Londrina, PR; \\ (3) $\mathrm{SCT}$ - CNPq - ON, CP 23002, 20921 Rio de Janeiro, RJ, BRASIL.
}

\begin{abstract}
The $\mathrm{H}_{2} \mathrm{O}$ maser source in $\mathrm{G} 305.8-0.2$ was first detected by Haynes et al. (1984). Inspection of the Southern Hemisphere radio surveys did not show any strong compact HII region or water maser placed nearby this source. The IRAS point source catalog (2.0) shows a strong source in the direction of the maser. We analysed the temporal variation and line profile of the $\mathrm{H}_{2} \mathrm{O}$ maser emission and discussed the nature of its associated IRAS source. These source characteristics seem compatible with the hypothesis that the maser is associated with a protostellar disk around an early-type star of spectral type 07 to 09 surrounded by a spherical she1l of gas and dust.
\end{abstract}

\section{Equipment and observations}

Radio observations were made using the 13.7 meter antenna of the Itapetinga Radio Observatory. In the first period (Jun-Dec84) weekly observations were made using a filter bank consisting of 46 channels of $100 \mathrm{kHz}$. During the second period (Sep90-Jun91) monthly observations were made using an acousto-optical spectrometer with 1000 channels of $40 \mathrm{kHz}$. A $1000 \mathrm{~K}$ receiver was used in both periods.

\section{Results and Interpretation}

During the first period the flux density of the $-26 \mathrm{kms}^{-1}$ line doubled, reaching a maximum in 8 days $(1500 \mathrm{Jy})$, returning to preburst level after 20 days. Assuming a kinematic distance of $2 \mathrm{kpc}$ Vilas Boas et al. (1991) computed that the isotropic luminosity of the maser was $10^{30} \mathrm{erg} \cdot \mathrm{s}^{-1}$. Its temporal evolution could be explained by a pulse of energy $E_{O}$ produced by the heating source radiating diffusivelly through the gas cloud. This maser line is saturated. The pumping changes as radiation diffuses through the cloud. To fit the model proposed by Burke et al.(1978), a pulse with energy of $10^{41}$ erg should be injected in the cloud. If we assume that this energy is supplied to the cloud in a time scale of a day and the emission is anisotropic (Alcock and Ross, 1985) it implies a heating source with 
maximum luminosity of $10^{39} \mathrm{erg}_{\bullet} \mathrm{s}^{-1}$. The Plank's fit to the IRAS fluxes suggests the existence of two shells, one of $130 \mathrm{~K}$ with $3 \times 10^{-12}$ sr and other of $50 \mathrm{~K}$ with $10^{-9}$ sr. The nfrared integrated luminosity of $2 \times 10^{4} \mathrm{~L}_{\mathrm{O}}$, together with the maser luminosity, suggests the presence of an 07 to 09 ZAMS star. If the temporal evolution of the maser indicates the heating source activity, then the 20 solar mass star we are dealing with is younger than $10^{5}$ yr (Panagia,1973). The absence of radio continuum implies that the circumstellar ionized gas is extremely thick at radio wavelengths or that the circumstellar dust is optically thick within the Lyman continuum, suppressing the Fig. 1 - Schematic representation formation of an HII region.

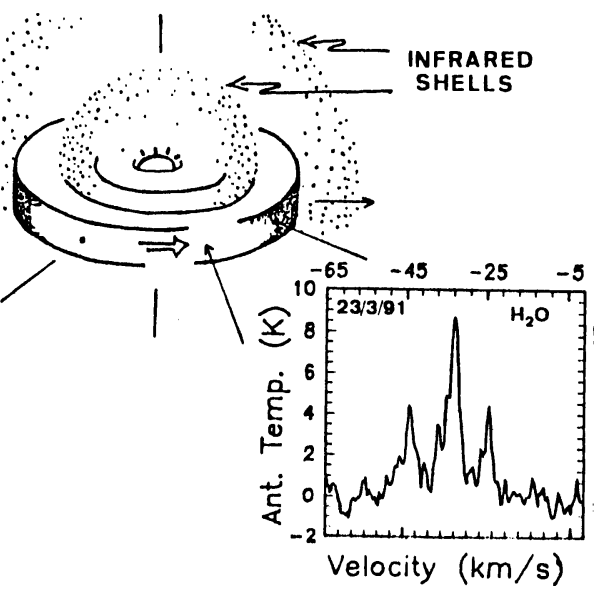
Specially during the second period the profiles observed were in good agreement with line profiles from protostellar disks (Grining and Gregor'ev, 1980). Figure 1 shows the $\mathrm{H}_{2} \mathrm{O}$ maser emission observed in Mars, 91. If we assume that the disk is in gravitational equilibrium with the central star, the velocity of the lateral lines suggests that the emission is originated in a region of the disk placed $10^{15}$ to $4 \times 10^{15} \mathrm{~cm}$ from the central star, what is in agreement with Elmegreen and Morris (1979) for maser emission from a 20 solar mass star surrounded by a one solar mass disk. Only between June and September, 84 we have observed a weak line with a velocity of $-56 \mathrm{kms}^{-1}$. Its intensity changed in a time scale of days and its microwave emission rate was $0.5 \mathrm{~s}^{-1}$. This suggests that the emission is unsaturated. Acceleration of cloudlets by the wind of the central star (Elmegreen and Morris, 1979) could explain the high velocity of this emission. Radio continuum and CO line observation of this region at millimeter wavelengths are of extreme importance to study the envelope of this star looking for bipolar outflow structures and continuum emission from cold dust.

\section{References}

Alcock, C. \& Ross, R.R. (1985) Ap. J., 290, 433.

Burke, B.F., Giufrida, T.S. \& Haschick, A.D. (1978) Ap. J•, 266, L21.

Elmegreen, B.G. \& Morris, M. (1979) Ap. J., 299, 593.

Grinin, V.P., \& Gregor'ev, S.A. (1983) Sov. Astron. Lett., 9, 244.

Haynes, R.F., Roger, R.S., Forster, B.J., Robinson, R., Batchelor, A. \& Wellington, K.J. (1984), IAU Circular no 3943.

Vilas Boas, J.W.S., Scalise Jr., E. \& Sanzovo, G.C. (1991) Astr. J., in press. 